\title{
Rad54 is required for the normal development of male and female germ cells and contributes to the maintainance of their genome integrity after genotoxic stress
}

\author{
S Messiaen ${ }^{1,2,3}$, A Le Bras ${ }^{1,2,3}$, C Duquenne $e^{1,2,3}$, V Barroca ${ }^{1,2,3}$, D Moison ${ }^{1,2,3}$, N Déchamps ${ }^{4}$, M Doussau ${ }^{5}$, A-L Bauchet ${ }^{5}$, \\ M-J Guerquin ${ }^{1,2,3}$, G Livera ${ }^{1,2,3}$, J Essers ${ }^{6,7,8}$, R Kanaar $^{6,7}$, R Habert $^{1,2,3}$ and J Bernardino-Sgherri ${ }^{, 1,2,3}$
}

Rad54 is an important factor in the homologous recombination pathway of DNA double-strand break repair. However, Rad54 knockout (KO) mice do not exhibit overt phenotypes at adulthood, even when exposed to radiation. In this study, we show that in Rad54 KO mouse the germline is actually altered. Compared with the wild-type (WT) animals, these mice have less premeiotic germ cells. This germ cell loss is found as early as in E11.5 embryos, suggesting an early failure during mutant primordial germ cells development. Both testicular and ovarian KO germ cells exhibited high radiation sensitivity leading to a long-term gametogenesis defect at adulthood. The KO female germline was particularly affected displaying decreased litter size or sterility. Spermatogenesis recovery after irradiation was slower and incomplete in Rad54 KO mice compared with that of WT mice, suggesting that loss of germ stem cell precursors is not fully compensated along the successive rounds of spermatogenesis. Finally, spermatogenesis recovery after postnatal irradiation is in part regulated by glial-cell-line-derived neurotrophic factor (GDNF) in KO but not in irradiated WT mice, suggesting that Sertoli cell GDNF production is stimulated upon substantial germ cell loss only. Our findings suggest that Rad54 has a key function in maintaining genomic integrity of the developing germ cells. Cell Death and Disease (2013) 4, e774; doi:10.1038/cddis.2013.281; published online 15 August 2013

Subject Category: Experimental Medicine

Spermatogenic and oogenic lineages are established in the mid-gestation mouse fetus. Primordial germ cells (PGC) first appear at embryonic day (E) 7.0, in the extra-embryonic mesoderm at the base of the allantois. Then, they migrate into the hindgut endoderm at E8.0 and travel along the dorsal mesentery by E9.5 to finally start the colonization of the genital ridges at E10.5-E11.5. ${ }^{1,2}$ Commitment of germ cells to male or female development occurs by E12.5.

Female germ cells enter meiosis at E13.5 and then arrest in diplotene stage at birth. Meiosis completion occurs during postnatal life. In contrast, murine male germ cells undergo a period of quiescence from E13.5 to birth and differentiate into spermatogonial populations including a pool of cells with spermatogonial progenitor cell properties. ${ }^{3}$ By 8 to 10 days after birth meiosis is initiated and the first spermatozoa appear 35 days after birth (P35). This period constitutes the so called first round of spermatogenesis whose regulation is thought to be different from continuous spermatogenesis in adults. ${ }^{4}$ During this period, Sertoli cells, which serve as supporting cells, gradually mature and change in morphology and in their functional aspects. ${ }^{5}$ At the begining of puberty, they cease dividing and form tight junctions, creating the blood-testis barrier and providing a specialized and protected environment within the testis.

Germ cells have to preserve their genomic integrity to ensure intact genetic information to the next generation. However, knowledge of how germ cells are maintained is still limited, ${ }^{6,7}$ particularly with regards to DNA repair capacities in fetal or neonatal premeiotic germ cells. ${ }^{8,9}$

DNA damage can be induced by chemical or physical agents. In case of ionizing radiation a wide spectrum of DNA lesion types has been reported. Among these lesions, DNA double-strand breaks (DSBs) are considered as the most dangerous for cell survival. DSBs can be repaired by two major pathways according to the cell cycle phase at the time of

${ }^{1}$ CEA, DSV/iRCM/SCSR/LDG, laboratory of Development of the Gonads, Unit of Stem Cells and Radiation, Fontenay-aux-Roses F-92265, France; ${ }^{2}$ Université Paris Diderot, Sorbonne Paris Cité, Fontenay-aux-Roses F-92265, France; ${ }^{3}$ INSERM, U967, Fontenay-aux-Roses F-92265, France; ${ }^{4}$ CEA, DSV/IRCM, Flow cytometry Shared Resource, Fontenay-aux-Roses F-92265, France; ${ }^{5} \mathrm{CRC}$ MIRCen CEA/INSERM Laboratoire de Modélisation des Biothérapies-Bât. 61, Fontenay-aux-Roses 92265, France; ${ }^{6}$ Department of Cell Biology and Genetics, Cancer Genomics Center, Erasmus MC, PO Box 2040, 3000 CA Rotterdam, The Netherlands; ${ }^{7}$ Department of Radiation Oncology, Erasmus MC, PO Box 2040, 3000 CA Rotterdam, The Netherlands and ${ }^{8}$ Department of Vascular Surgery, Erasmus MC, PO Box 2040,3000 CA Rotterdam, The Netherlands

*Corresponding author: J Bernardino-Sgherri, DSV/iRCM/SIGRR/LRIG, Centre CEA, BP6, 18 route du panorama, F-92265, Fontenay-aux-roses, France. Tel: + 331465480 04; Fax: + 331465499 06; E-mail: jacqueline.bernardino@ cea.fr

Keywords: Rad54; germ cells; ovary; testis; development; radiation

Abbreviations: GDNF, glial-cell-line-derived neurotrophic factor; SSEA1, stage-specific embryonic antigen 1; SCO, Sertoli cell only; Gy, gray; PLZF, promyelocytic leukemia zinc finger protein; Ngn3, neurogenin3; POU5F1, POU domain class 5 transcription factor 1; CD49, cluster of differentiation 49; CDH1, cadherin 1; FITC, fluorescein isothiocyanate; PE, phycoerythrin; UV, ultraviolet; PMSF, phenylmethanesulfonylfluoride; DDX4, DEAD/H box polypeptide 4; TUNEL, terminal deoxynucleotidyl transferase dUTP Nick End Labeling; BrdU, bromodeoxyuridine; DAPI, 4',6'-diamidino-2-phénylindole

Received 17.5.13; revised 25.6.13; accepted 02.7.13; Edited by G Raschellá 
exposure. Non homologous end joining, which consists of the simple ligation of DNA strands, is the fastest mechanism and is functional in all phases of the cell cycle. ${ }^{10}$ Homologous recombination (HR) is functional only in $\mathrm{S}$ and $\mathrm{G} 2$ phases as a homologous DNA template is needed, usually the sister chromatid. This is also the reason why HR is also considered as the most reliable pathway to achieve high fidelity cellular DNA repair. ${ }^{11}$

Among the proteins that are critically involved in $H R$, RAD51 is the most important one as its deficiency leads to early embryonic lethality. However, RAD54, which is a protein that directly binds RAD51, does not seem to be essential to HR as Rad54 mouse mutants are viable, fertile and insensitive to radiation. ${ }^{12}$ Nevertheless, HR is compromised in Rad54 mutant cells. In contrast to adults, Rad54 knockout (KO) embryos (E3.5) were found to be highly radiosensitive. ${ }^{13}$ Interestingly, Rad54-deficient mouse embryonic stem cells but not embryonic fibroblasts were shown to be radiosensitive. This suggests that Rad54 role in radiation protection may vary according to the cell type considered and the age at irradiation. As Rad54 is preferentially expressed in proliferating cells, ${ }^{14}$ and because germline establisment involves several waves of high testicular cell proliferation activity, we hypothesized that efficient HR could be more critical during embryonic and early postnatal germ cell development than during adult gametogenesis.

To test this hypothesis, we studied both male and female germline development in Rad54 KO mice. We also exposed the mice to $\gamma$-rays during embryonic development and at the time of spermatogonial stem cell establishment and followedup spermatogenesis recovery through adulthood.

\section{Results}

Three-month-old Rad54-deficient male and female mice show decreased spermatogonial populations and reduced ovarian reserve, respectively. Fifteen-week-old $\mathrm{KO}$ mice showed no overt alterations in testicular weight and histology although spermatogonial populations were reduced (Supplementary Figure S1). At the same age, KO female mouse ovaries showed a dramatic decrease in the number of primordial follicles compared with wild-type (WT) mice (Figure 1) despite no difference in their litter size $(7.1 \pm 0.6$ for WT versus $5 \pm 1$ for KO). These data show that Rad54 is critical in the generation of the mouse ovarian reserve and contributes, although to a lesser extent, to adult spermatogonial population maintainance.

Surprisingly, at earlier stages no significant changes were observed among spermatogonial, meiotic and post-meiotic cells between KO and WT mice, except at P14 where the undifferentiated spermatogonial population was increased in KO mice (Supplementary Figures S1 and S7a). Interestingly, the amount of testicular glial-cell-line-derived neurotrophic factor (GDNF) was increased in KO testes only at this age in agreement with its role as a regulator of spermatogonial proliferation $^{15-17}$ (Supplementary Figure S7b). This suggests that Rad54 has a particular role in the regulation of spermatogonial population amount during the first round of spermatogenesis.

\section{Germ cell number but not Sertoli cell number depends} on Rad54 during fetal life. At all ages analyzed, the number of germ cells was reduced in both $\mathrm{KO}$ male and female mice compared with WT (Figures 2a-f). However, we failed to detect any change in the E14.5 KO Sertoli cell population (Figure 2d). A small increase in germ cell apoptotic rate was detected in KO compared with WT at P4 (Figure 2c and Supplementary Figure S2). In contrast, no increase in either ectopic or apoptotic germ cells was detected in KO compared with WT E11.5 embryos (Figure 2e).

Interestingly, Rad54 was expressed at about 10-fold higher levels in germ compared with fetal Sertoli cells purified by flow cytometry (Supplementary Methods and Supplementary Figure S3). This difference was also detected between P3 germ and Sertoli cells but was less marked than in the fetus (Supplementary Figure S3). In E13.5 female gonads, levels of
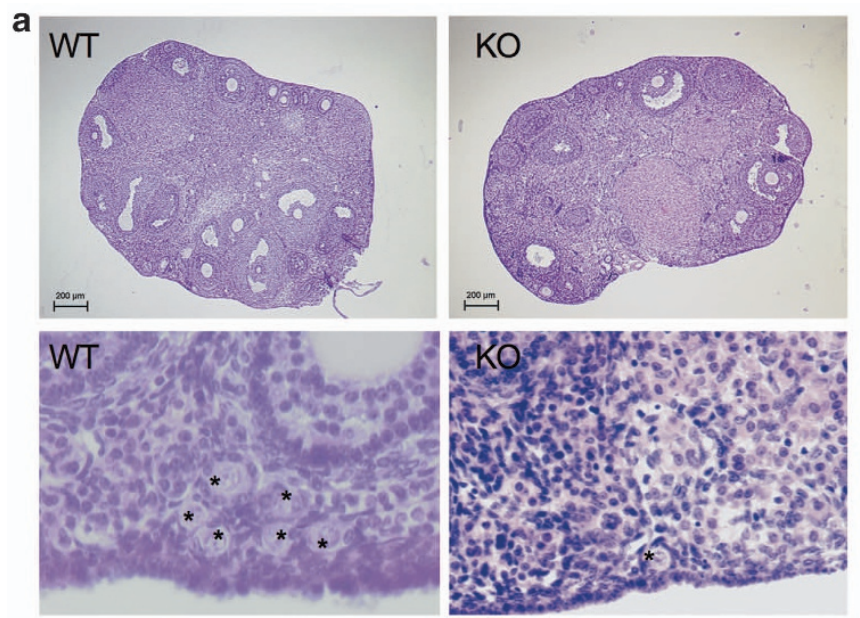

b

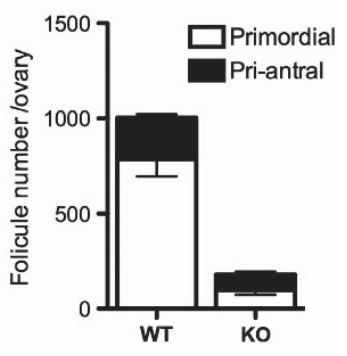

Figure 1 Rad54 KO ovaries have reduced ovarian reserve. (a) Hematoxylin and eosine staining of 3-month-old WT and KO ovaries. Lower panel represents an enlarged picture ( $\times 40$ objective) showing primordial follicles $\left(^{*}\right)($ b) Follicle counts show depletion in 'pri-antral' follicles (represents the sum of primary, secondary, pre-antral and antral follicles) and particularly the primordial follicle pool that determines the female fertility life span $(n=5)$ 

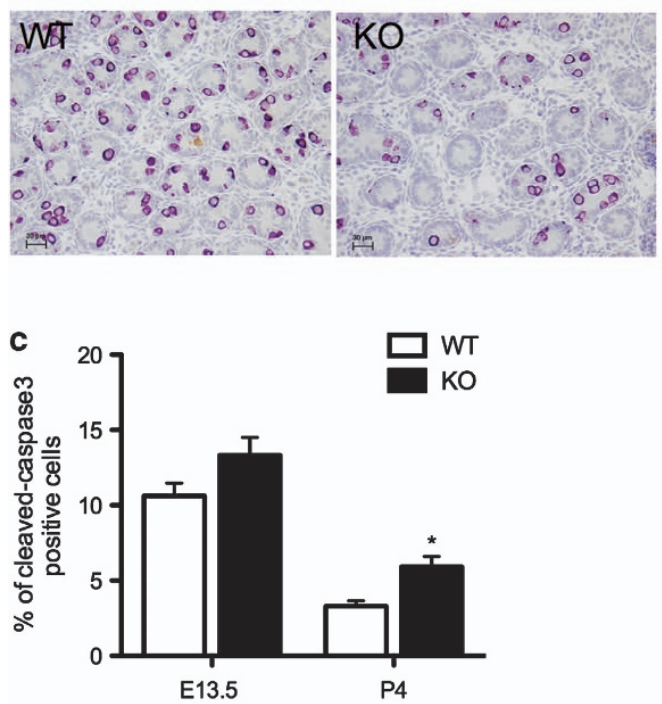

e

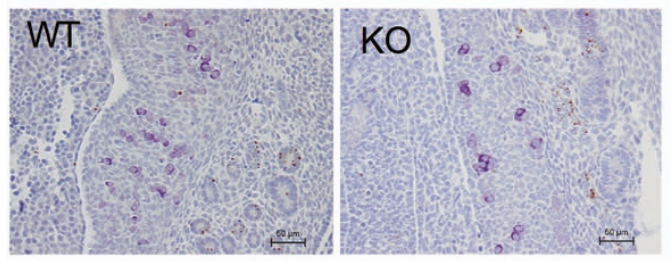

b

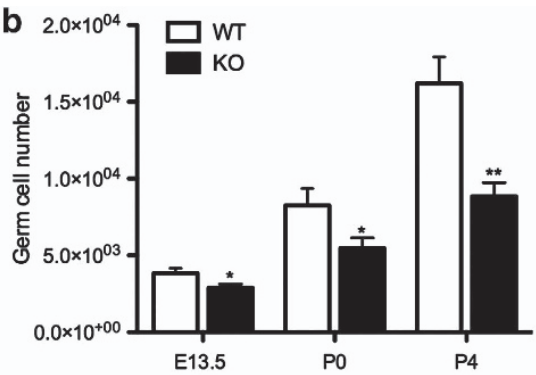

d
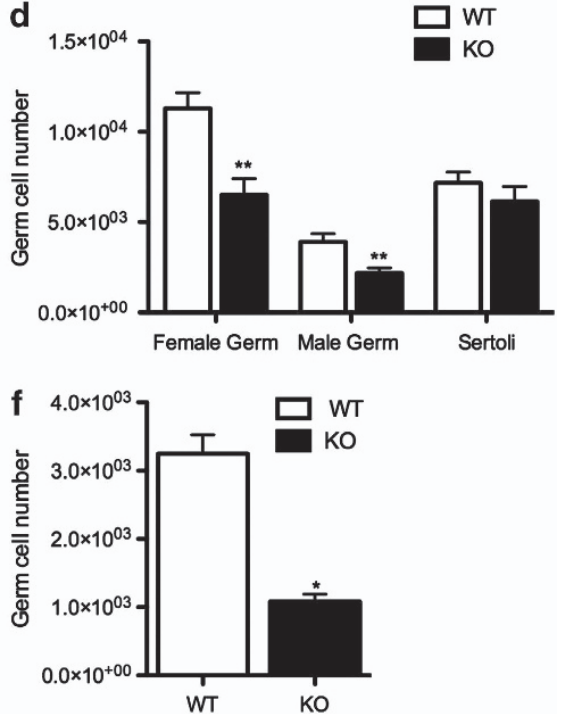

Figure 2 Rad54 is required for normal germ cell development. (a) Immunohistochemical staining of DDX4 (purple) and cleaved-caspase-3 (brown) in P4 testes cross sections from WT and Rad54 KO mice. (b) Total germ cell count (DDX4-positive cells) analysis in both type of mice at different ages. (c) Percentage of apoptotic cells (cleaved caspase-3 positive cells) in E13.5 and P4 testes from WT and Rad54 KO mice. (d) Flow cytometric analysis of the germ cell and somatic cell absolute numbers in E14.5 WT and KO mice, using beads as internal control. (e) Testis cross section from E11.5 WT and KO stained for DDX4 (purple) and terminal deoxynucleotidyl transferase dUTP nick end labeling (TUNEL) (brown). (f) PGC number in E11.5 fetuses counted on all sections of total embryo. At least five animals per group were analyzed except for E11.5 $(n=3-4)$ embryos. Data are presented as mean \pm S.E.M. Statistically significant differences between WT and KO are indicated by asterisks $\left({ }^{*} P<0.05 ;{ }^{* *} P<0.01\right)$

Rad54 mRNA in stage-specific embryonic antigen 1 (SSEA1)positive cells (female premeiotic germ cells) were also higher than in SSEA1-negative cells (mostly ovarian somatic cells and some meiotic germ cells) (not shown).

Taken together, these data demonstrate that Rad54 is required for normal germ cell embryonic development and suggest that Rad54 is dispensable for the survival of somatic supporting cells of the gonads.

Fetal exposure to moderate dose of $\gamma$-rays has long-term deleterious effects on testis and ovary. Although Rad54 $\mathrm{KO}$ mice are fully fertile, ${ }^{12}$ the deficiency could affect the response to genotoxic stress. We thus asked whether Rad54 absence could affect mouse fertility after radiation exposure. A 2 Gy (gray) dose on KO E13.5 embryos was lethal at birth in 9 out of 10 litters. Clinical examination showed that irradiated KO newborns were smaller compared with irradiated WT and exhibited facial deformity. We killed the two surviving males at 15 weeks of age and found that they were hydrocephalic, had smaller organs and that their testes were completely devoid of germ cells, whereas the irradiated WT controls exhibited only $18.5 \%$ tubules devoid of germ cells (Sertoli cell only, (SCO)) (Figure 3).
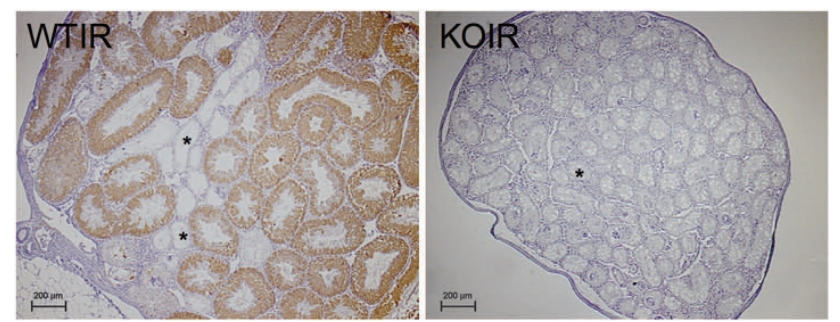

Figure 3 Fetal irradiation at 2 Gy of KO mice lead to male sterility at adulthood. Immunohistochemical staining of DDX4 on testis cross section of WT (left panel) and KO (right panel) irradiated mice. WT-irradiated testis exhibit $18.5 \%$ of SCO seminiferous tubules $\left({ }^{*}\right)$ compared with $100 \%$ SCO in irradiated KO testis

Because of the lethality of the $2 \mathrm{~Gy}$ dose delivered to KO fetuses, we analyzed both the short- (Figure 4) and the long-term (Figure 5) effects of fetal irradiation at 0.5 Gy. In WT-irradiated mice, there was a similar decrease in the number of gonocytes and that of oogonia (66 and 69\%, respectively) at $24 \mathrm{~h}$ after treatment. This was in contrast to KO-irradiated oogonia ( $80 \%$ decrease) that were slightly less radiosensitive than male gonocytes (94\% decrease) (Figure 4b). Using flow cytometric analysis, we also showed 

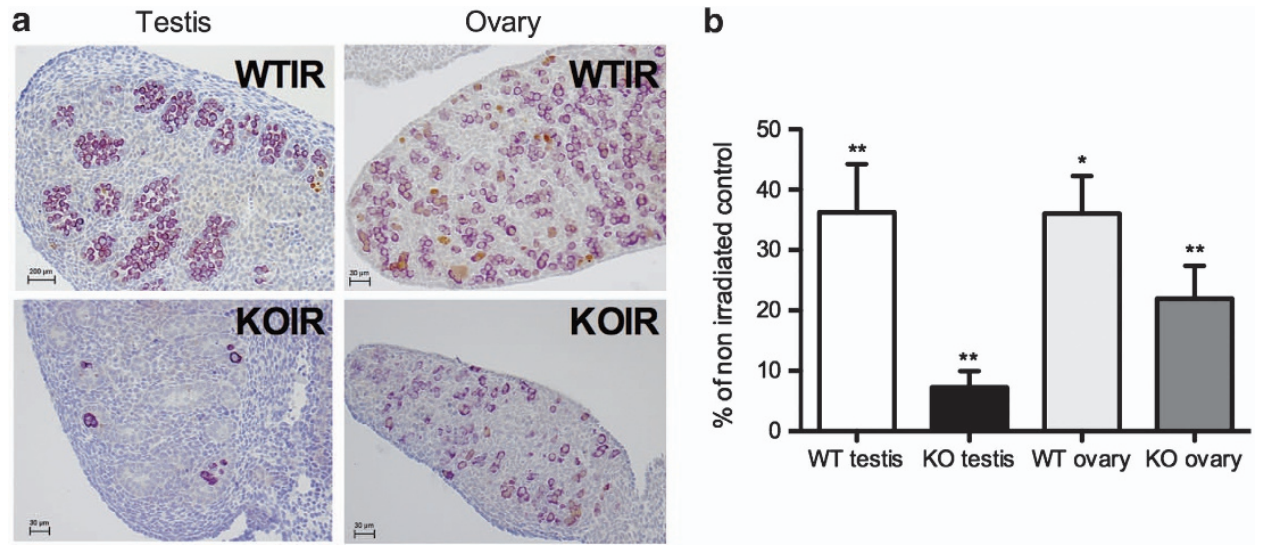

Figure 4 Both KO female and male E13.5 embryonic gonads are more radiosensitive than WT. (a) Immunohistochemical staining of DDX4 (purple) (left panels) or double staining of DDX4 (purple)/POU5F1 (brown) (right panels) of E14.5 gonad cross sections from mice irradiated $24 \mathrm{~h}$ before at 0.5 Gy. (b) Representation of premeiotic germ cell counts in the whole gonads. In testes, DDX4-positive cells were counted. In ovary, POU5F1 and DDX4-double positive cells (i.e., oogonia) were counted. Four to six animals per group were analyzed. Data are presented as the percentage of the corresponding non-irradiated control (mean \pm S.E.M.). Statistically significant differences between irradiated and non-irradiated gonads are indicated by asterisks $\left({ }^{\star} P<0.05 ;{ }^{*} P<0.01\right)$

that radiation strongly reduced $\mathrm{KO}$ germ cell numbers, whereas those of fetal Sertoli cells were not affected at all (Supplementary Figure S4), suggesting that Rad54 is dispensable for the survival of these cells after challenging with acute irradiation.

After fetal irradiation at $0.5 \mathrm{~Gy}$, all animals survived at least 3 months. Three-month-old KO-irradiated testes showed $47 \pm 3 \%$ SCO, whereas almost none SCO $(<0.1 \%)$ was observed in irradiated WT testes. Many KO-irradiated germ cell subpopulations were reduced when compared with the non-irradiated controls, whereas no change was found in irradiated WT testes compared with controls (Supplementary Figure S5).

From the stronger deleterous effect of Rad54 deletion on the female compared with the male germline, we inferred that exposure of E13.5 KO female fetuses to 0.5 Gy could lead to infertility at adulthood. At this dose, the ovarian histology was deeply altered in 15-week-old KO animals (Figure 5a). Interestingly, irradiated WT females ovaries had different size, both having similar amounts of follicles except for primordial follicles that were not found at all in the smallest ones (Figures $5 \mathrm{~b}$ and $\mathrm{c}$ ). In another experiment, three WT- and three KO-irradiated females (0.5 Gy at E13.5) were mated at the age of 3 months with healthy WT males. One out of three KO-irradiated females was sterile. The other two females exhibited a lower litter size compared with WT-irradiated females, which were all fertile (Figure 5d).

Altogether, these results show long-term stronger deleterous effect of acute fetal radiation exposure on both the female and the male Rad54 KO germlines compared with WT. Moreover, the moderate dose of $0.5 \mathrm{~Gy}$ was sufficient to sterilize KO but not WT-irradiated females.

Testicular regeneration in irradiated mice depends in part on their Rad54 status. Spermatogonial stem cells are established postnatally and could therefore allow spermatogenesis regeneration after both fetal and neonatal exposure to genotoxic agents. This could explain why similar alteration in fetal germ cell numbers lead to less severe outcome for male than for female mice gonads, as female fertility is determined by the pool of primordial follicles established during fetal life. Regeneration after either fetal or neonatal radiation exposure occurs around or before the end of the first round of spermatogenesis (P35). ${ }^{18}$

In an attempt to determine the kinetics of spermatogenesis regeneration after irradiation in both WT and KO mice, we searched for testis alteration at different age after irradiation (2Gy) at the time of spermatogonial stem cells establishment (P3). In both P3 WT and KO mice, almost all germ cells were proliferating ( $80.7 \pm 3.4 \%$ Ki67-positive germ cells for WT and $75.3 \pm 6.4 \%$ for KO; $P=0.7 ; n=4$ ) reflecting their differentiation into spermatogonia. ${ }^{19}$ Whatever the age of analysis, KO-irradiated testes exhibited lower testicular weight (Supplementary Figure S6a) and higher rate of SCO tubules compared with irradiated WT testes (Supplementary Figure S6b). We estimated using regression analysis that the reduction by half of SCO percentage took about twice as long in irradiated KO than in irradiated WT.

Altogether, these data are consistent with higher long-term radiation sensitivity of Rad54-deficient neonatal testis. Moreover, spermatogenesis recovery efficiency and kinetics after P3 irradiation depend in part on the Rad54 status.

Kinetics of spermatogenesis recovery of P3-irradiated mice depends mostly on the extent of initial germ cell killing. To gain insight into the role of Rad54 in spermatogenesis recovery after irradiation at P3, we quantified germ cell subpopulations of the testes at different ages. Within the first $24 \mathrm{~h}$ after irradiation, less germ cells were found in $\mathrm{KO}$ compared with WT-irradiated mice (27 and $57 \%$ of nonirradiated controls, respectively), confirming the higher radiation sensitivity of Rad54-deficient P3 germ cells (Figure 6a). Concomittantly, the amount of GDNF was upregulated only in KO-irradiated mice (Figure 6b).

Almost all meiotic subpopulations of WT-irradiated mice fully recovered at $\mathrm{P} 105$, while in KO-irradiated mice they remained about $30 \%$ of the non-irradiated level (Supplementary Figure S7c). Total spermatogonial 
a

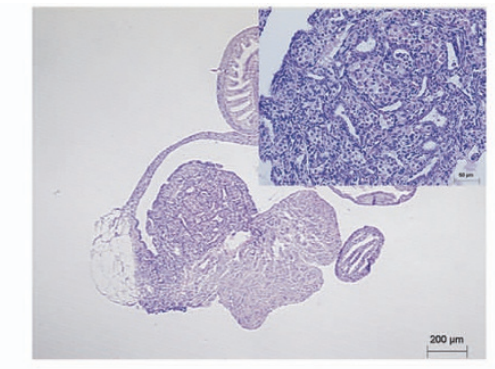

b

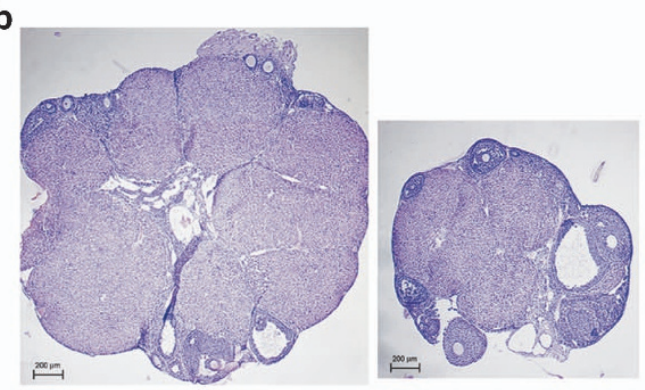

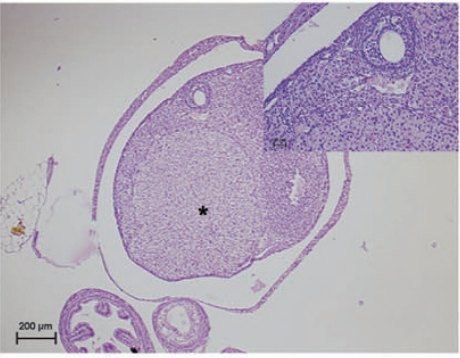

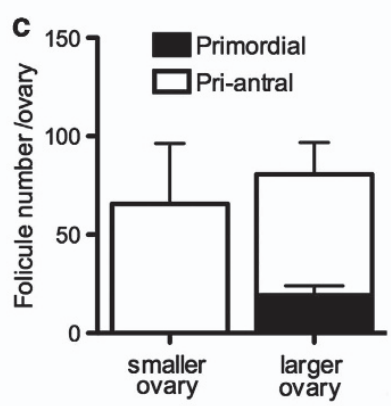

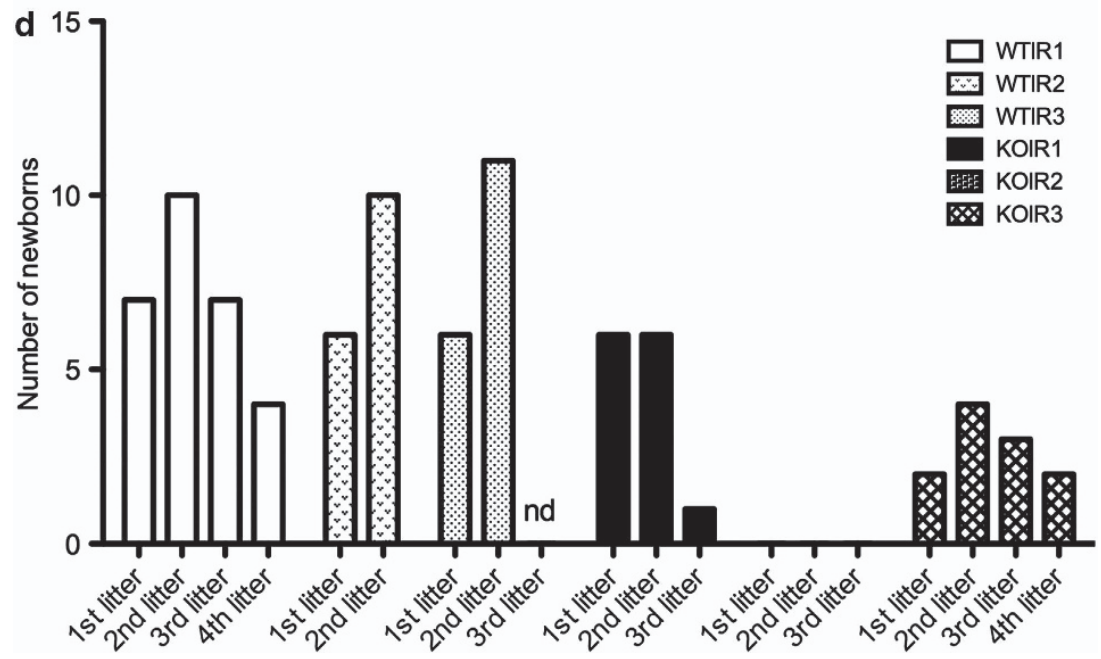

Figure 5 Fetal irradiation of female KO mice may lead to sterility or more precocious ovarian failure than irradiation of WT females. (a) Two examples of ovarian cross sections from two different 3-month-old females irradiated with $0.5 \mathrm{~Gy}$ at E13.5. On the left, the ovarian organ structure is disorganized. As shown in the inset, tubule-like structures are formed. These structures have been associated with differentiation of the somatic granulosa cell in Sertoli like cells upon severe depletion in germ cells. ${ }^{36}$ On the right, a cross section of an ovary showing hyperplasia of a corpus luteus ( $\left.{ }^{*}\right)$ and in the inset a remaining follicle. (b) Each irradiated WT female mouse exhibited a smaller ovary than the other one as shown in these cross sections. (c) Follicle counts revealed the absence of primordial follicles in the smallest ovary. Data are represented as mean \pm S.E.M; $n=5$. (d) Adult WT females irradiated as fetuses were all fertile $(n=3)$ and showed similar litter size when continuously in presence of a WT non-irradiated male for several months. On the contrary KO-irradiated females $(n=3)$ were sterile or still fertile but with smaller litter size either since the first litter or later

populations in WT-irradiated mice were recovered at P35 (Figure 6c). This spermatogonial regeneration involved upregulation of the most undifferentiated subpopulation (highly stained for promyelocytic leukemia zinc finger protein, (PLZF)) at P14 and increased spermatogonial differentiation until P22 (increased KIT + and reduced KIT- populations at P22) (Figure 6d). The amount of GDNF did not change until P14, suggesting GDNF-independent regulation of spermatogonial proliferation in these mice (Figure 6b). Dramatic spermatogonial depletion occurred in $\mathrm{KO}$-irradiated mice with $90 \%$ SCO tubules at P7 (Supplementary Figure S6b) and upregulated GDNF compared with non-irradiated control (Figure $6 \mathrm{~b})$. Although differentiating spermatogonia $(\mathrm{KIT}+$ ) were severely reduced at P14 (Figure 6c), proliferation of Plzf-high undifferentitated spermatogonia increased at P14 leading to higher numbers of this subpopulation at P22 (Figure 6d). At P35, spermatogonial populations increased but remained about $30 \%$ of the non-irradiated control at P105 (Figure $6 \mathrm{c}$ ).

Altogether, these data show that spermatogonial population regeneration is slower and less efficient in KO than in WT $\mathrm{P} 3$-irradiated mice and that this regeneration relies on GDNF upregulation only in $\mathrm{KO}$ testes.

\section{Discussion}

In this study, we show for the first time the specific requirement of Rad54 for normal germ cell development, and this in both genders. Absence of this gene leads to a 

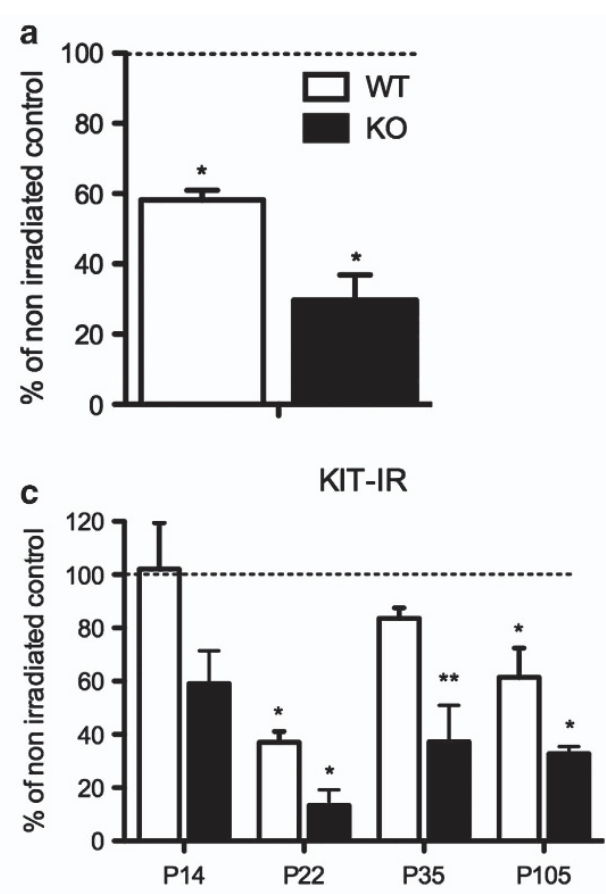
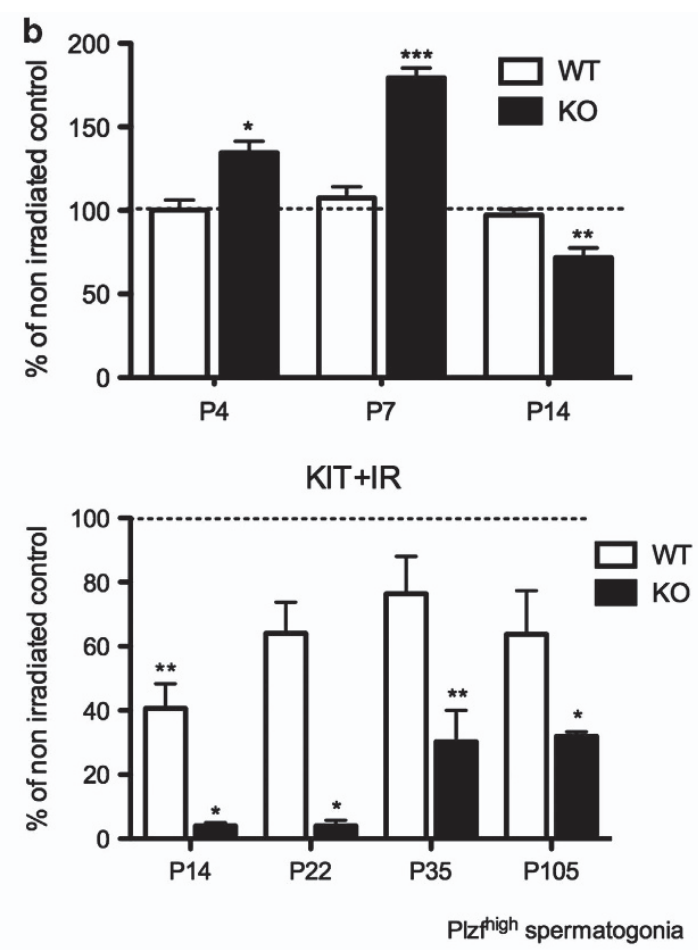

d
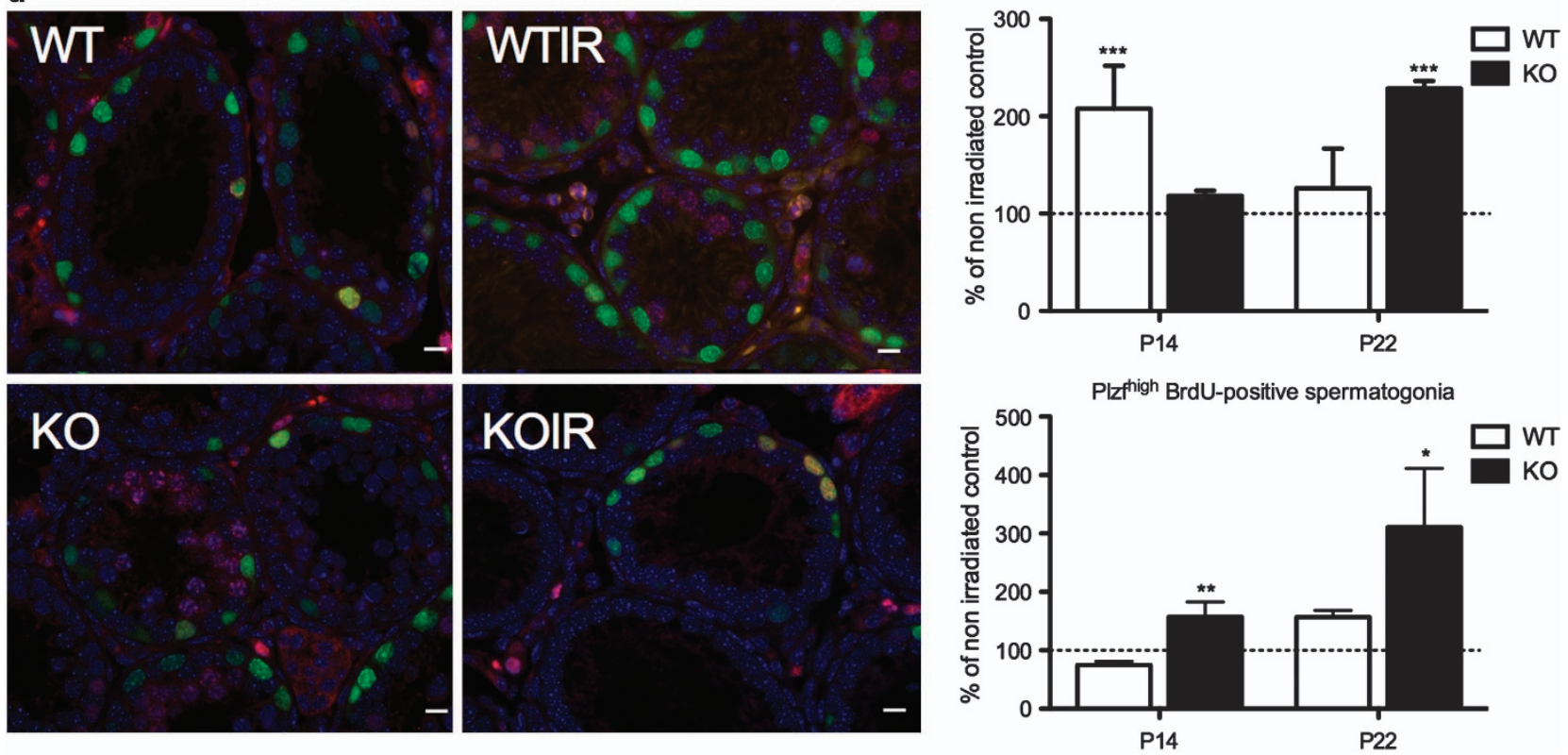

Figure 6 Follow-up of spermatogonial germ cell subpopulations after P3 irradiation. (a) Immunohistological analysis of the germ cell numbers (DDX4-positive cells) in testes from WT and KO mice $24 \mathrm{~h}$ after irradiation at P3 (2 Gy). Data are presented as the percentage of the non-irradiated control (mean \pm S.E.M; $n=4)$. (b) Testicular GDNF production is induced within the first $24 \mathrm{~h}$ after irradiation of KO P3 mice only $(n=5)$. (c) Flow cytometric analysis of the spermatogonial subpopulation absolute numbers at different ages. Four to six animals per group were analyzed. (d) Immunohistochemical analysis of the $\mathrm{Plzf}^{\text {high }}$-spermatogonial subpopulation per seminiferous tubule cross section (PLZF: green; BrdU: red; DAPI: blue) $(n=3)$. Statistically significant differences between irradiated and non-irradiated gonads are indicated by asterisks $\left({ }^{\star} P<0.05\right.$; $\left.{ }^{* \star} P<0.01 ;{ }^{* \star *} P<0.001\right)$

significant loss of premeiotic germ cells during fetal, early postnatal and adult life that was concomittant in some instances with a modest increase in apoptotic rate. This loss may be related to early defect in Rad54 KO PGC development (before E11.5). Rad54 is mostly expressed during S and G2 phases of the cell cycle. ${ }^{14}$ Therefore, it is tempting to speculate that the absence of Rad54 could somehow specifically alter PGC cycle progression before E11.5, leading to a reduced number of germ cells at later stages as shown in other KO mouse models. ${ }^{20,21}$

We also found other mild but significant phenotypes in $\mathrm{KO}$ postnatal germ cells. P14, but not P22 or P35, testes had a higher number of spermatogonial populations and a higher amount of GDNF. This suggests the existence at P14 of a 
mechanism regulating the number of spermatogonia during the first launch of meiosis. This mechanism could involve detection of a defect in the amount of germ cells that stimulates Sertoli cell production of GDNF, which in turn stimulates proliferation of spermatogonial progenitors. At these ages, the number of Sertoli cells per tubule was the same in KO and WT (our unpublished data). This suggests that, as in the embryonic gonad, premeiotic postnatal germ cell number seems to be more dependent on Rad54 than the amount of Sertoli cells. In addition, the lack of difference in the number of WT and KO spermatogonia in P22 and P35 testes could be explained by the fact that by P21 a physiological apoptotic wave occurs to ensure a proper ratio between maturing germ and Sertoli cells. ${ }^{22}$ Therefore, it could well be that, at P14, the supernumary undifferentiated spermatogonia produced upon GDNF stimulation would further be eliminated during the physiological apoptotic wave.

In sexually mature $\mathrm{KO}$ mice, however, the undifferentiated spermatogonia were $28 \%$ less abundant than in WT mice. This is in contrast with the lack of difference at P35 but can be explained by the heterogeneity of the newborn germ cell population according to whether they express or not $\mathrm{Ngn} 3$ (neurogenin3). ${ }^{3}$ The Ngn3-negative germ cells directly differentiate to generate the first spermatozoa at P35 (that is, the first round of spermatogenesis), whereas the Ngn3-positive spermatogonial lineage is responsible for the generation of the initial pool of spermatogonial stem cells from which future spermatogonial stem cells and progenitor spermatogonia are derived during steady-state spermatogenesis in adulthood. Therefore, our results suggest that Rad54 deficiency alters both the Ngn3-negative and positive lineages but that compensatory mechanisms are more efficient during the first round of spermatogenesis.

As previously described by Essers et al., ${ }^{12} \mathrm{KO}$ mice fertility was not affected in spite of the important depletion in premeiotic germ cells that we have shown in this study. Li et $a^{23}$ found a $60 \%$ loss of primordial follicles in 3-week-old female Rad54 KO mutant mice compared with WT. In the present study, we found an even higher loss (90\%) in 15-week-old KO female mice in comparison with WT. No overt histological alteration was observed in male adult testis at least until 9 months of age. This suggests that loss of $40 \%$ of fetal or neonatal premeiotic germ cells is not sufficient to alter either male or female mouse fertility.

The most deleterous phenotypes of Rad54 KO mice that were evident so far include high radiation sensitivity of E3.5 embryos exposed to $4 \mathrm{~Gy} .{ }^{24}$ Here we report that a dose of 2 Gy gamma rays at E13.5 is lethal at birth for most Rad54 KO mice, while WT-irradiated control counterparts developed normally and were fully fertile at adulthood. This shows that Rad54 is absolutely required for mouse survival after a threshold of genotoxic stress during mid-gestation. Moreover, the two $\mathrm{KO}$ males that survived $2 \mathrm{~Gy}$ irradiation at E13.5 exhibited strong alterations in behavior, hydrocephally and were completely sterile. This underscores the importance of Rad54 for both brain ${ }^{25}$ and germline development following genotoxic stress.

E13.5 irradiation at 0.5 Gy could lead to complete sterility of $\mathrm{KO}$ female mice and when mice were still fertile, a strong reduction in litter size resulted compared with irradiated
WT mice. This suggests that irradiation of pregnant mice with constitutive deeply altered ovarian reserve may lead to premature ovarian failure. Interestingly, WT-irradiated females also exhibited significant depletion of follicles characterized by the particular small size of one ovary that exhibited no primordial follicles at all. To our knowledge, this has not been reported before ${ }^{26}$ and may depend on rodent species, genetic background, age at irradiation or dose. Similar irradiation conditions which lead to $66 \%$ of germ cells loss in WT male, did not have a detrimental effect on spermatogenesis at adulthood as shown by no difference in the germ cell subpopulations compared with non-irradiated control. This suggests that this loss is compensated later during postnatal establishment of mature spermatogenesis involving waves of germ cell proliferation. ${ }^{27}$ Irradiation of KO mice, however, lead to strong depletion in most of the germ cell subpopulations of the adult testis eventhough mice are still fertile. We previously showed that irradiated mice with only $5 \%$ sperm counts of nonirradiated control can be still fully fertile. ${ }^{18}$ We can assume from our data that either a threshold of fetal germ cell loss is needed to trigger long-term deleterious effects on spermatogenesis or, that Rad54 is involved in spermatogenesis recovery.

We favor the first hypothesis since in postnatally irradiated WT mice, the kinetics of testicular weight recovery parallels that of KO-irradiated mice. Furthermore, testicular weight of irradiated WT mice does not reach that of non-irradiated control, even 9 months after irradiation. Another point is that a twofold higher loss of germ cells after postnatal irradiation doubles the time required for KO-irradiated mice to repopulate seminiferous tubules. This observation fits with the observation that in adult irradiated mice, the interval needed for the recovery of fertility is directly proportional to the level of killing of the spermatogonial stem cells. ${ }^{28}$ Upregulation of testicular GDNF levels after irradiation occurs only in $\mathrm{KO}$ mice. These data are in agreement with GDNF mRNA being strongly upregulated after X-irradiation of adult WT mice with 3-4 Gy. ${ }^{29}$ However, as GDNF is not upregulated in WT newborn mice irradiated with 2 Gy our results suggest that GDNF is only required when there is a large depletion of germ cells. Although GDNF has been demonstrated to be the master regulator of spermatogonial proliferation, ${ }^{15}$ it is likely that recovery from moderate loss of germ cell after genotoxic stress may be regulated by Sertoli cells via GDNF-independent pathways or by autonomous germ cell mechanisms.

Interestingly, whereas irradiated $\mathrm{KO}$ fetal testes were almost completely depleted of germ cells, we found no significant effect on Sertoli cell number. Rad54 KO mouse embryonic fibroblasts (MEFs) were not found to be more radiosensitive to $\gamma$-rays than WT MEF, ${ }^{12}$ whereas embryonic neural stem cells require Rad54 for survival after irradiation. ${ }^{25}$ This emphasizes the tissue-specificity of DNA repair pathways dependent on Rad54 during embryonic development after genotoxic stress.

In conclusion, we show that in the developing gonad, Rad54 is critical for maintaining premeiotic germ cell viability after endogenous and exogenous DNA damage. In constrast, the germ supporting cells (Sertoli cells) do not require Rad54 for their survival even after irradiation. Therefore, mammalian germ and somatic cells have developed distinct mechanisms 
to maintain their genomic integrity as recently reported for C. elegans. ${ }^{30}$

\begin{abstract}
Materials and Methods
Mice. The mice used were homozygous transgenic Rad54 ${ }^{+/+}$(WT) and Rad54 ${ }^{-1-}$ (KO) mice on a C57BL/6J background. Mice were bred and maintained according to the guidelines for the care and use of laboratory animals of the French Ministry of Agriculture.
\end{abstract}

Irradiation. Mice were whole-body exposed to $\gamma$-rays from a ${ }^{137} \mathrm{Cs}$ source (IBL 637; CIS bio International, Gif sur Yvette, France) at a dose rate of $0.6 \mathrm{~Gy} / \mathrm{min}$ and killed at different time points after exposure.

BrdU injection. Intraperitoneal injection of $50 \mathrm{mg} / \mathrm{kg}$ bromodeoxyuridine (BrdU; Sigma-Aldrich, Lyon, France) was delivered to 14- and 22-day-old (P14 and P22) mice. Mice were then killed $3 \mathrm{~h}$ after treatment.

Histology. Mouse adult ovaries (15 weeks old) were fixed in Bouin's, embedded in paraffin, serially sectioned at $5 \mu \mathrm{m}$ and stained for hematoxylin and eosin. For oocyte quantification, every fifth section was scored for the presence of the following classes of oocytes: primordial, primary, secondary, pre-antral and antral, using the criteria previously described.$^{31}$ Only those with a visible nucleus were counted.

Fetal, postnatal and adult testes were fixed overnight in $10 \%$ neutral formalin at $4{ }^{\circ} \mathrm{C}$ before being embeded in paraffin as previously described. ${ }^{18}$

Immunohistochemistry. Immunohistochemistry on fetal, postnatal and adult gonads was performed as previously described. ${ }^{18}$ Primary antibodies used in this study were rabbit polyclonal anti-DEAD/H box polypeptide 4; (DDX4; $1: 400$, Abcam, Paris, France), mouse monoclonal anti-Ki67 (1:50, BD Biosciences, Le Pont de Claix, France), goat polyclonal anti-GATA-4 (1:200, Santa Cruz Biotechnology/ CliniSciences, Nanterre, France), rabbit polyclonal anti-cleaved caspase-3 (1:100, Cell Signaling/Merck Millipore, Saint-Quentin-en-Yvelinnes), rabbit polyclonal antiPLZF (1:100, Santa Cruz Biotechnology), mouse monoclonal anti-BrdU $(1: 200$, Roche diagnostics, Meylan, France), mouse anti-POU domain class 5 transcription factor 1 (POU5F1; 1:50, Santa Cruz Biotechnology).

Primary antibodies were revealed using IMPRESS kit (Vector Laboratories/ Clinisciences) and counterstained with hematoxylin. For immunofluorescence detection, primary antibodies were detected with anti-mouse-Alexa488 (1:1000, Invitrogen, Saint Aubin, France), anti-rabbit-Alexa594 (1:1000, Invitrogen) and counterstained with DAPI $\left(4^{\prime}, 6^{\prime}\right.$-diamidino-2-phénylindole; vector Laboratories/ Clinisciences).

ISEL staining was performed using the Apoptag peroxidase kit (MP Biomedicals, Illkirch, France) as described. ${ }^{32}$

Fluorescent images were captured using an Olympus AX70 epifluorescent microscope (Olympus France SAS, Rungis, France) equipped with charge-coupled camera (Princeton Instruments, Trenton, NJ, USA) and IPLab software (Scanalytics, Fairfax, VA, USA).

Cell counts after immunohistochemistry. Germ cells were counted in one of five sections equidistantly distributed along the gonad, using Histolab software (Microvision Instruments, Evry, France).

For counting number of POU5F1-positive PGCs in both the female and male gonads of E11.5, serial sagittal sections were made from each entire fetus. POU5F1-positive cells in all serial sections were counted and total number of the positive cells per gonad was scored. ${ }^{20}$

Cell staining and flow cytometric analysis. Fetal testes and ovaries devoid of mesonephros or P3 testes were incubated in HBSS containing $1 \mathrm{mg} / \mathrm{ml}$ collagenase I (Serva Electrophoresis, Heidelberg, Germany) and $0.02 \mathrm{mg} / \mathrm{ml}$ DNase I (Invitrogen) for $15 \mathrm{~min}$ at $37^{\circ} \mathrm{C}$ in a shaking water bath. Every $5 \mathrm{~min}$, the fragments were resuspended with a pipette. After complete digestion, cells were centrifuged for $5 \mathrm{~min}$ at $300 \mathrm{~g}$ and resuspended in HBSS. Immunolabelling was performed using CD9, SSEA1, CD49f (cluster of differentiation $49 \mathrm{f}$ ), Thy1 or/and cadherin 1 (CDH1) antibodies coupled with fluorochromes (BD Biosciences): E13.5 testicular cells, were incubated for 30 in $2 \%$ BSA/HBSS(w/v) at room temperature before incubation with anti-CD9 biotinylated (BD Biosciences) for
$30 \mathrm{~min}$. After washes in $2 \% \mathrm{BSA} / \mathrm{HBSS}(\mathrm{w} / \mathrm{v})$, cells were incubated with anti-CD49f$\mathrm{PE}$ (phycoerythrin) and anti-biotin-APC (BD Biosciences) for $30 \mathrm{~min}$. After two washes in HBSS, cells were resuspended in $2 \% \mathrm{BSA} / \mathrm{HBSS}(\mathrm{w} / \mathrm{v})$ before cell sorting. E13.5 ovarian cells were also incubated for $1 \mathrm{~h}$ in $2 \% \mathrm{BSA} / \mathrm{HBSS}(\mathrm{w} / \mathrm{v})$ before incubation with anti-SSEA1-PE. P3 testicular cells were incubated with antiThy1-APC, anti-CDH1-PE and CD49f-FITC (fluorescein isothiocyanate) for at least 30 min. After two washes with HBSS, cells were incubated in $2 \% \mathrm{BSA} / \mathrm{HBSS}(\mathrm{w} / \mathrm{v})$ before cell sorting.

Cell sorting was performed using a three-laser MoFlo (Cytomation, Fort Collins, CO, USA). A Coherent Innova 90-5 argon-ion laser, emitting $200 \mathrm{~mW}$ at $488 \mathrm{~nm}$ was used to measure scatter and to excite FITC (520/40) and PE (585/40) fluorescences. A Coherent Innova 308-ultraviolet argon-ion laser, emitting $100 \mathrm{~mW}$ at $351-365 \mathrm{~nm}$ was used to excite $\mathrm{HO} 33258(0.5 \mu \mathrm{g} / \mathrm{ml}$-Sigma-Aldrich) was added to exclude dead cells. APC fluorescence was exited at $635 \mathrm{~nm}$ and fluorescent signal collected through a $661 \mathrm{~nm}$ bandpass filter. PE fluorescence was exited at $488 \mathrm{~nm}$ and fluorescent signal collected through a $585 \mathrm{~nm}$ bandpass filter. FITC fluorescence was excited et $488 \mathrm{~nm}$ and fluorescent signals collected through a $520 / 40$ bandpass filter. Germ and Sertoli cell populations were identified and sorted in RLT buffer (Qiagen, Courtaboeuf, France) for RNA extraction and expression analyses (Supplementary Methods).

Pre-pubertal and adult testicular single-cell suspensions, immunolabellings and flow cytometric analyses were performed as previously described. ${ }^{33,34}$ For fetal, pubertal and adult testicular subpopulations, absolute counts were established using flow cytometry and fluorescent beads (TruCOUNT, BD Biosciences) and a LSRII cytometer (BD Biosciences).

Reverse transcription and real-time PCR. Total RNA was extracted from sorted germ cells using the RNeasy micro kit (Qiagen) according to the manufacturer's instruction. Total RNAs were reverse transcribed using High Capacity kit (Qiagen). Real-time PCR reactions were carried out according to the manufacturer's instructions in an ABI Prism 7900 Sequence Detection system (Applied Biosystems, Saint Aubin, France).

The following probes were used: Actb ( $\beta$-actin) Mm00607939_s1, Rad54l Mm00485522_m1 (designed on request by Applied Biosystems). mRNAs were quantified using the TaqMan Gene Expression Master Mix (Applied Biosystems) in a total volume of $20 \mathrm{ml}$. Samples were heated for $10 \mathrm{~min}$ at $95^{\circ} \mathrm{C}$, followed by 45 cycles of $15 \mathrm{~s}$ at $95^{\circ} \mathrm{C}$ then $1 \mathrm{~min}$ at $60^{\circ} \mathrm{C}$. Each sample was run in duplicate for every primer/probe combination.

Quality of sorted cell fractions was verified using RT-QPCR with sets of primers for cell type specific markers as previously described. ${ }^{35}$

GDNF quantification. Total testicular protein extracts were obtained using a FastPrep system (three runs for $25 \mathrm{~s}$ at $6.5 \mathrm{~m} / \mathrm{s}$ and $5 \mathrm{~min}$ incubation in ice between each run) and Lysis Matrix D tubes (MP biomedicals). The lysis buffer contained $137 \mathrm{~mm} \mathrm{NaCl}, 20 \mathrm{~mm}$ Tris (pH8), 1\% NP-40, 10\% glycerol, $1 \mathrm{~mm}$ phenylmethanesulfonylfluoride, protease cocktail (Roche diagnostics), phosphostop (Roche diagnostics). After centrifugation, the whole or a fraction of the supernatant was monitored for GDNF quantification using the enzyme-linked immunosorbent assay Emax Immunoassay according to the suppliers instructions (Promega, Lyon, France). On each 96-well plates, three standard curves were performed.

Statistical analysis. Results are presented as means \pm S.E.M. Statistical analyses were performed with Prism 5 (GraphPad software, La Jolla, USA) applying either the Student's $t$-test or non-parametric Mann-Whitney $U$-test. ${ }^{*} P<0.05 ;{ }^{*} P<0.01 ;{ }^{* * *} P<0.001$.

\section{Conflict of Interest}

The authors declare no conflict of interest.

Acknowledgements. We are gratefull to Sophie Tourpin for technical advices on real-time PCR experiments, Nelly Frydman for constant support, Pierre Fouchet for fruitfull discussions and suggestions, and Pablo Radicella for critical reading of the manuscript. We also thank V Neuville, S Leblay, J Tilliet and C Joubert for animal care. This work was supported by Electricite de France (EDF). We thank the IRCM Flow Cytometry Shared Resource that was established by equipment Grants from DIM-Stem Pôle, INSERM, Fondation ARC pour la Recherche sur le Cancer, and CEA. 
1. Bendel-Stenzel M, Anderson R, Heasman J, Wylie $C$. The origin and migration of primordial germ cells in the mouse. Semin Cell Dev Biol 1998; 9: 393-400.

2. McLaren A. Primordial germ cells in the mouse. Dev Biol 2003; 262: 1-15.

3. Yoshida S, Sukeno M, Nakagawa T, Ohbo K, Nagamatsu G, Suda T et al. The first round of mouse spermatogenesis is a distinctive program that lacks the self-renewing spermatogonia stage. Development 2006; 133: 1495-1505.

4. Ebata KT, Zhang X, Nagano MC. Male germ line stem cells have an altered potential to proliferate and differentiate during postnatal development in mice. Biol Reprod 2007; 76 841-847.

5. Petersen $\mathrm{C}$, Soder $\mathrm{O}$. The sertoli cell-a hormonal target and 'super' nurse for germ cells that determines testicular size. Hormone Res 2006; 66: 153-161.

6. Jaroudi S, SenGupta S. DNA repair in mammalian embryos. Mutat Res 2007; 635: 53-77.

7. Rube CE, Zhang S, Miebach N, Fricke A, Rube C. Protecting the heritable genome: DNA damage response mechanisms in spermatogonial stem cells. DNA Repair 2011; 10: 159-168.

8. Xu G, Spivak G, Mitchell DL, Mori T, McCarrey JR, McMahan CA et al. Nucleotide excision repair activity varies among murine spermatogenic cell types. Biol Reprod 2005; 73: 123-130.

9. Hajkova P, Jeffries SJ, Lee C, Miller N, Jackson SP, Surani MA. Genome-wide reprogramming in the mouse germ line entails the base excision repair pathway. Science 2010; 329: 78-82.

10. Mahaney BL, Meek K, Lees-Miller SP. Repair of ionizing radiation-induced DNA doublestrand breaks by non-homologous end-joining. Biochem J 2009; 417: 639-650.

11. Jeggo PA, Geuting V, Lobrich $M$. The role of homologous recombination in radiationinduced double-strand break repair. Radiother Oncol 2011; 101: 7-12.

12. Essers J, Hendriks RW, Swagemakers SM, Troelstra C, de Wit J, Bootsma D et al. Disruption of mouse RAD54 reduces ionizing radiation resistance and homologous recombination. Cell 1997; 89: 195-204.

13. Essers J, van Steeg H, de Wit J, Swagemakers SM, Vermeij M, Hoeijmakers JH et al. Homologous and non-homologous recombination differentially affect DNA damage repair in mice. EMBO J 2000; 19: 1703-1710.

14. Kanaar R, Troelstra C, Swagemakers SM, Essers J, Smit B, Franssen JH et al. Human and mouse homologs of the Saccharomyces cerevisiae RAD54 DNA repair gene: evidence for functional conservation. Curr Biol 1996; 6: 828-838

15. Meng X, Lindahl M, Hyvonen ME, Parvinen M, de Rooij DG, Hess MW et al. Regulation of cell fate decision of undifferentiated spermatogonia by GDNF. Science 2000; 287: 1489-1493.

16. Oatley JM, Avarbock MR, Brinster RL. Glial cell line-derived neurotrophic factor regulation of genes essential for self-renewal of mouse spermatogonial stem cells is dependent on Src family kinase signaling. J Biol Chem 2007; 282: 25842-25851.

17. Johnston DS, Olivas E, DiCandeloro P, Wright WW. Stage-specific changes in GDNF expression by rat Sertoli cells: a possible regulator of the replication and differentiation of stem spermatogonia. Biol Reprod 2011; 85: 763-769.

18. Forand A, Messiaen S, Habert R, Bernardino-Sgherri J. Exposure of the mouse perinatal testis to radiation leads to hypospermia at sexual maturity. Reproduction 2009; 137: 487-495.

19. Culty M. Gonocytes, the forgotten cells of the germ cell lineage. Birth Defect Res C Embryo Today 2009; 87: 1-26.

20. Abe K, Naruse C, Kato T, Nishiuchi T, Saitou M, Asano M. Loss of heterochromatin protein 1 gamma reduces the number of primordial germ cells via impaired cell cycle progression in mice. Biol Reprod 2011; 85: 1013-1024.

21. Lutzmann M, Grey C, Traver S, Ganier O, Maya-Mendoza A, Ranisavljevic N et al. MCM8 and MCM9-deficient mice reveal gametogenesis defects and genome instability due to impaired homologous recombination. Mol Cell 2012; 47: 523-534.
22. Rodriguez I, Ody C, Araki K, Garcia I, Vassalli P. An early and massive wave of germinal cell apoptosis is required for the development of functional spermatogenesis. EMBO J 1997; 16: 2262-2270.

23. Li XC, Bolcun-Filas E, Schimenti JC. Genetic evidence that synaptonemal complex axial elements govern recombination pathway choice in mice. Genetics 2011; 189: 71-82.

24. Essers J, Hendriks RW, Wesoly J, Beerens CE, Smit B, Hoeijmakers JH et al. Analysis of mouse Rad54 expression and its implications for homologous recombination. DNA Repair 2002; 1: 779-793.

25. Rousseau L, Etienne O, Roque T, Desmaze C, Haton C, Mouthon MA et al. In vivo importance of homologous recombination DNA repair for mouse neural stem and progenitor cells. PloS One 2012; 7: e37194.

26. Mazaud S, Guigon CJ, Lozach A, Coudouel N, Forest MG, Coffigny H et al. Establishment of the reproductive function and transient fertility of female rats lacking primordial follicle stock after fetal gamma-irradiation. Endocrinology 2002; 143: 4775-4787.

27. Filipponi D, Hobbs RM, Ottolenghi S, Rossi P, Jannini EA, Pandolfi PP et al. Repression of kit expression by Plzf in germ cells. Mol Cell Biol 2007; 27: 6770-6781.

28. Meistrich ML, Hunter NR, Suzuki N, Trostle PK, Withers HR. Gradual regeneration of mouse testicular stem cells after exposure to ionizing radiation. Radiat Res 1978; 74: 349-362.

29. Ventela S, Makela JA, Kulmala J, Westermarck J, Toppari J. Identification and regulation of a stage-specific stem cell niche enriched by Nanog-positive spermatogonial stem cells in the mouse testis. Stem cells 2012; 30: 1008-1020.

30. Vermezovic J, Stergiou L, Hengartner MO, d'Adda di Fagagna F. Differential regulation of DNA damage response activation between somatic and germline cells in Caenorhabditis elegans. Cell Death Differ 2012; 19: 1847-1855.

31. Myers M, Britt KL, Wreford NG, Ebling FJ, Kerr JB. Methods for quantifying follicular numbers within the mouse ovary. Reproduction 2004; 127: 569-580.

32. Forand A, Bernardino-Sgherri J. A critical role of PUMA in maintenance of genomic integrity of murine spermatogonial stem cell precursors after genotoxic stress. Cell Res 2009; 19: 1018-1030.

33. Bastos H, Lassalle B, Chicheportiche A, Riou L, Testart J, Allemand I et al. Flow cytometric characterization of viable meiotic and postmeiotic cells by Hoechst 33342 in mouse spermatogenesis. Cytometry A 2005; 65: 40-49.

34. Lassalle B, Bastos H, Louis JP, Riou L, Testart J, Dutrillaux B et al. 'Side Population' cells in adult mouse testis express Bcrp1 gene and are enriched in spermatogonia and germinal stem cells. Development 2004; 131: 479-487.

35. Forand A, Fouchet P, Lahaye JB, Chicheportiche A, Habert R, Bernardino-Sgherri J. Similarities and differences in the in vivo response of mouse neonatal gonocytes and spermatogonia to genotoxic stress. Biol Reprod 2009; 80: 860-873.

36. Guigon CJ, Coudouel N, Mazaud-Guittot S, Forest MG, Magre S. Follicular cells acquire sertoli cell characteristics after oocyte loss. Endocrinology 2005; 146: 2992-3004.

(c) (1) (2) Cell Death and Disease is an open-access journal published by Nature Publishing Group. This work is licensed under a Creative Commons Attribution-NonCommercialShareAlike 3.0 Unported License. To view a copy of this license, visit http://creativecommons.org/licenses/by-nc-sa/3.0/

\section{Supplementary Information accompanies this paper on Cell Death and Disease website (http://www.nature.com/cddis)}

